\title{
Case Report 16
}

Ramiro Hernandez, M.D. ${ }^{1}$, Andrew K. Poznanski, M.D. ${ }^{1 *}$, and Robert Hensinger, M.D. ${ }^{2}$

${ }^{1}$ Division of Pediatric Radiology and

${ }^{2}$ Section of Orthopedic Surgery, C.S. Mott Children's Hospital, The University of Michigan Medical Center,

Ann Arbor, Michigan, USA
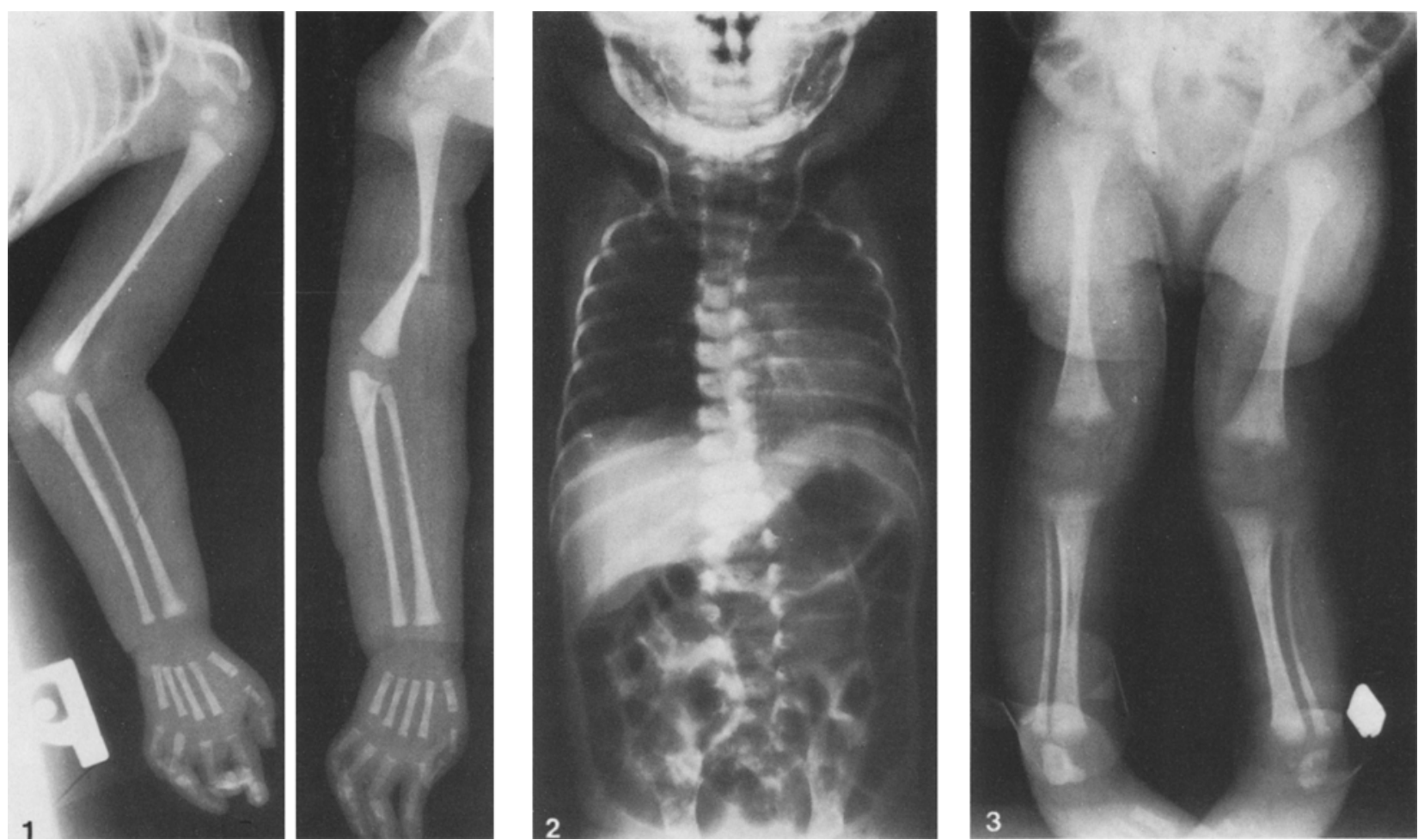

Fig. 1. Observe the extremely long, thin bones of the arms and hands. Contractures of the fingers are noted. A fracture just below the middle third of the right humerus is present

Fig. 2. Note the extremely thin ribs and clavicles

Fig. 3. Observe the delayed maturation in this one-week-old child, with very small distal femoral ossification centers and absent tibial epiphyses. This disharmony may be compared with the upper extremities in Figure 1 where the humeral centers are clearly ossified. Observe the diminution in muscle mass in the upper and lower extremities (also see Fig. 1)

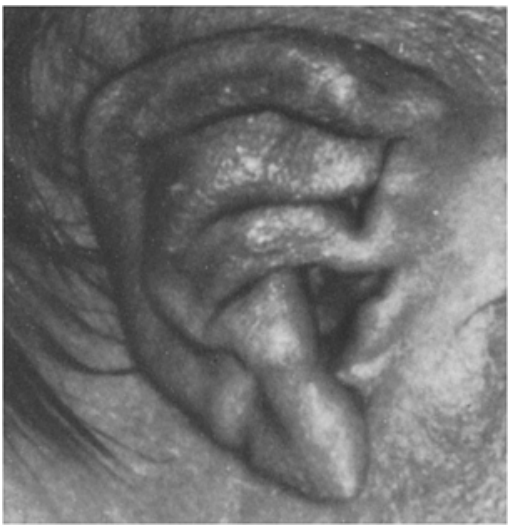

Fig. 4. A photograph of the ear demonstrates it to be crumpled, with flattening of the helix

\section{History}

This one-week-old 2.4-kg baby was the product of a 38-week gestation of a 15 -week-old black, unmarried mother. Multiple contractures were noted to involve the hips, knees, elbows, wrists, and all the proximal interphalangeal joints, except the left index finger. Motion was relatively normal beyond the flexion contractures. Examination of the eyes showed no abnormality.

* Presented by Dr. Andrew K. Poznanski at the 3rd Annual Meeting of the International Skeletal Society in Montreal, Canada, September 10th-12th, 1976

Address reprint requests to: A.K. Poznanski, M.D., Division of Pediatric Radiology, C.S. Mott Children's Hospital, The University of Michigan Medical Center, Ann Arbor, MI 48104, USA 


\section{Diagnosis: Congenital Contractural Arachnodactyly (C.C.A.)}

The differential diagnosis includes arthrogryposis, Marfan syndrome, Stickler syndrome and osteogenesis imperfecta.

\section{Discussion}

This entity was first recognized by Beals and Hecht in 1971. It features multiple congenital contractures, long thin bones (dolichostenomelia), thin fingers (arachnodactyly), scoliosis, and ear abnormalities, which include a flattened helix with a crumpled appearance. Congenital heart disease may be present. The contractures may regress with time. Variability in the expression of the disorder exists. In some cases significant decrease in muscle mass and subcutaneous tissues (as in this infant) may occur. A documented inheritance is not available in the present case.

Patients with C.C.A. were initially reported as Mafan syndrome with arthrogryposis. In fact, Marfan's original patient most likely represented congenital contractural arachnodactyly, rather than what is known as Marfan syndrome today. In defining the entity in 1971, Beals reported two additional patients and reviewed the literature, where he found 12 kindreds whose findings were consistent with the diagnosis of C.C.A. Since that time additional cases have been reported.

C.C.A. may be differentiated from Marfan syndrome by the presence of contractures and the absence of lenticular dislocation, as well as the typical cardiac and aortic lesions generally present in Marfan syndrome. In C.C.A., the joint deformities are congenital, symmetrical and maximal at birth, while in Marfan syndrome they are usually absent at birth but may be progressive during childhood. General joint laxity, common in Marfan syndrome, is not present in C.C.A. Abnormalities of the ear are not part of the Marfan syndrome.

The idiopathic form of arthrogryposis multiplex congenita must be differentiated from C.C.A. In many of the old case reports of arthrogryposis, other entities such as C.C.A., Larsen syndrome and others may have been included. Extremely thin bones and fractures also have been observed in arthrogryposis. The dolichostenomelia and arachnodactyly, however, are not particularly characteristic of the usual idiopathic arthrogryposis. The joint contractures of arthrogryposis typically result in profound limitation of joint motion, both in flexion and extension, whereas in C.C.A. the contracture primarily limits extension of the joint, but full flexion is still possible.

Stickler syndrome (hereditary arthopthalmopathy) is characterized by dolichostenomelia, but differs from C.C.A. in that it is associated with hyperextensibility of joints, congenital myopia, retinal detachment, flat facies, and occasionally the Pierre-Robin syndrome.

Osteogenesis imperfecta may be considered in the differential diagnosis, since slender bones and fractures may be present in both conditions. However, the contractures and dolichostenomelia in C.C.A. are not a hallmark of osteogenesis imperfecta. The other manifestations of osteogenesis, such as blue sclerae, are not part of the presentation of C.C.A.

Differentiation of C.C.A. from Marfan syndrome, as well as the other entities listed, is important from the point of view of prognosis and genetic counselling.

\section{References}

1. Beals, R.K., Hecht, F.: Congenital contractural arachnodactyly. A heritable disorder of connective tissue. J. Bone Jt Surg. 53A, 987 (1971)

2. Hecht, F., Beals, R.K.: "New" syndrome of congenital contractural arachnodactyly originally described by Marfan in 1896 . Pediatrics 49, 574 (1972)

3. Lipson, E.H., Viseskul, C., Herrmann, J.: The clinical spectrum of congenital contractural arachnodactyly. A case with congenital heart disease. Z. Kinderheilk. 118, 1 (1974)

4. Marfan, A.B.: Un cas de déformation congenitale des quatre membres, plus prononcée aux extremités, caractérisée par l'allongement des os, avec un certain degré d'amincissement. Bull. Mém. Soc. Méd. d'Hôp. Paris 13, 220 (1896)

5. Passarge, E.: A syndrome resembling congenital contractural arachnodactyly. Birth Defects: Orig. Art. Series, no. 6, 11, 53 (1975)

6. Poznanski, A.K., La Rowe, P.C.: Radiographic manifestations of the arthrogryposis syndrome. Radiology 95, 353 (1970) 